\title{
CAPITULO 23
}

\section{OS EFEITOS DO TREINAMENTO MUSCULAR INSPIRATÓRIO EM PACIENTES EM DESMAME DA VENTILAÇÃO MECÂNICA}

\author{
Daniel da Silva Gomes ${ }^{1}$, Diego Gomes da Silva ${ }^{2}$, Marinalva Ferreira Silva ${ }^{3}$ \\ Lorenna Valeria da Cruz Lima Dorneles ${ }^{4}$, Carla de Jesus dos Santos Vieira ${ }^{5}$, Maiza \\ Vieira Duarte Ribeiro ${ }^{6}$, Danielle de Brito Rodrigues ${ }^{7}$, Rosalice Campos de Sousa ${ }^{8}$, \\ Amália Ribeiro Gonçalves de Vasconcelos ${ }^{9}$, Suelen Gomes da Silva ${ }^{10}$, Emanuel Macedo \\ Costa $^{11}$, Ângela Letícia Silva Lima ${ }^{12}$ \\ ${ }^{1}$ Centro Universitário Santo Agostinho, (mariferreira2503@gmail.com) \\ ${ }^{2}$ Centro Universitário Santo Agostinho, (dieh.gs21@gmail.com) \\ ${ }^{3}$ Centro Universitário Santo Agostinho, (danielsgomes@gmail.com) \\ ${ }^{4}$ Centro Universitário Santo Agostinho, (lorenlimafisio@gmail.com) \\ ${ }^{5}$ Centro Universitário Santo Agostinho, (carlasantos061998@gmail.com) \\ ${ }^{6}$ Centro Universitário Santo Agostinho, (maizaribeiro47@gmail.com) \\ Centro Universitário Santo Agostinho, (danyeh97@gmail.com) \\ ${ }^{8}$ Centro Universitário Santo Agostinho, (rosalice.127@gmail.com) \\ ${ }^{9}$ Centro Universitário Santo Agostinho, (amaliargv@hotmail.com) \\ ${ }^{10}$ Centro Universitário Santo Agostinho, (emanuelmacedoc@gmail.com) \\ ${ }^{11}$ Centro Universitário Santo Agostinho, (lucianenppm@yahoo.com.br) \\ ${ }^{12}$ Centro Universitário Santo Agostinho, (angelaslima@gmail.com)
}

\section{Resumo}

Objetivo: Avaliar os efeitos do Treinamento Muscular Inspiratório (TMI) no desmame de pacientes em uso da ventilação mecânica. Métodos: Trata-se de um estudo clínico analítico; intervencionista; descritivo; longitudinal; coorte e controlado. Resultados: Pode-se observar que todos apresentaram critérios de elegibilidade, semelhança inicial entre grupos, análise de intenção de tratamento, comparação entre grupos e medidas de precisão e variabilidade. Em apenas um não houve distribuição aleatória e acompanhamento adequado. Conclusão: Os efeitos do treinamento muscular inspiratório em pacientes em desmame da ventilação mecânica se mostraram de forma bastante positiva em pacientes.

Palavras-chave: Cicatrização; Fototerapia; Recursos naturais; Fisioterapia.

Área Temática: Temas transversais - Outros. 
E-mail do autor principal: danielsgoms@gmail.com

\section{INTRODUÇÃO}

A respiração é um processo mecânico, automático, rítmico e regulado pelo sistema nervoso central. O sistema respiratório é responsável pelas trocas gasosas (Hematose), ele também auxilia na regulação da temperatura corporal e na manutenção do $\mathrm{pH}$ do sangue. É constituído pelo nariz, cavidade nasal, faringe, laringe, traquéia, brônquios e pulmões. $\mathrm{O}$ diafragma é o principal músculo da inspiração, é suprido pelos nervos frênicos a partir dos segmentos cervicais C3, C4 e C5 e constituído por fibras estriadas, com características próprias por ter maior constituição de fibras vermelhas ou oxidativas e, assim, mais resistentes à fadiga, diferindo dos demais músculos estriados esqueléticos do corpo (NUNES et al, 2019).

Diretamente ligada aos pulmões está à caixa torácica, dessa forma o ciclo respiratório exige sincronismo entre o pulmão e a musculatura respiratória. Movimentos respiratórios torácico e/ou abdominal, com a presença ou ausência da atividade de músculos acessórios, variáveis como volume corrente (VC), freqüência respiratória (FR), volume minuto (VE) e relação entre duração da inspiração e expiração, é o que compõe o padrão respiratório normal. O sistema respiratório é composto pelos músculos inspiratórios que são o diafragma e os intercostais externos, sendo o diafragma o principal músculo respiratório. Os músculos acessórios que são os músculos peitorais, maior e menor, o esternocleidomastóideo e os escalenos. E os músculos expiratórios que são abdominais e intercostais internos (NOZAWA et al, 2018).

Quando o individuo não tem capacidade de manter uma troca gasosa entre o organismo e a atmosfera de forma eficiente, dizemos que ele apresenta um quadro Insuficiência Respiratória Aguda (IRpA). É uma síndrome potencialmente grave e, por a respiração ter 3 fases: pulmonar, circulatória e mitocondrial, pode resultar de disfunções pulmonar, da parede torácica, dos músculos ventilatórios, de problemas cardíacos ou distúrbios mitocondriais, distúrbios do centro respiratório hipotalâmico (SOARES et al, 2018).

A fraqueza muscular desencadeada por longos períodos no ventilador mecânico está freqüentemente associada com a dificuldade ou falha no desmame posterior a traqueostomia, $o$ que pode retardar a alta hospitalar dos pacientes internados, além de aumentar os riscos de infecção pulmonar (DE OLIVEIRA et al, 2017).

Vários fatores podem estar relacionados ao acometimento muscular respiratório do paciente internado, que podem resultando em alterações funcionais e neurofisiológicas, com

\section{E - book Pesquisa e Tecnologia: Protagonismo e Inovações}


consequiente diminuição de força e resistência. A diminuição do aporte de substratos energéticos, por exemplo, que são necessários para um adequado funcionamento ou por uma alteração direta da fibra muscular, desde a condução do impulso neuromuscular até o intercâmbio gasoso. São músculos estriados que apresentam maiores fluxo sanguíneo, capacidade oxidativa e resistência à fadiga, quando comparados aos músculos esqueléticos. Entretanto, quando ocorre disfunção desta musculatura e a demanda ventilatória excede sua capacidade, podem ocorrer episódios de hipoventilação e hipoxemia (DE OLIVEIRA et al, 2017).

A manovacuometria é um teste por meio do qual a pressão inspiratória máxima (PImáx) e a pressão expiratória máxima (PEmáx) são obtidas, é simples, rápidos e não invasivo, que tem como objetivo auxiliar na avaliação muscular respiratória. È um teste que é realizado antes do treino respiratório, pois seus valores são necessários para traçar o protocolo de tratamento (DOS SANTOS et al, 2017).

Aproximadamente $30 \%$ a $40 \%$ dos pacientes internados em cuidados críticos usam como tratamento a ventilação mecânica. O tempo de permanência sob ventilação mecânica invasiva está associado à maior probabilidade de mortalidade. Em um levantamento sobre as características da VMI no Brasil, foi encontrado que a média de tempo de internamento em pacientes submetidos ao suporte ventilatório invasivo é de 16 dias (YEUNG, 2018; CORDEIRO, 2017).

Este estudo tem a finalidade de avaliar os efeitos do Treinamento Muscular Inspiratório (TMI) no desmame de pacientes em uso da ventilação mecânica.

\section{MÉTODO}

Trata-se de uma revisão integrativa, na qual foi realizado um levantamento bibliográfico nas bases de dados PUBMED, PEDro e Biblioteca Virtual em Saúde (BVS), utilizando as palavras-chaves disponíveis nos DECS: músculos respiratórios, treinamento de resistência, desmame e ventilação mecânica, inserindo os operadores boleanos AND e OR para tornar a pesquisa mais eficiente.

Foram considerados os seguintes critérios de inclusão: ensaios clínicos controlados e randomizados; ano de publicação no período de 2017 à 2021, que abordassem o Treinamento Muscular Inspiratório (TMI) em pacientes internados na Unidade de Terapia Intensiva (UTI) antes do processo de desmame do ventilador; pontuação mínima de 5 no escore da avaliação da qualidade metodológica da Escala PEDro. Como critérios de exclusão estão outras revisões de

\section{E - book Pesquisa e Tecnologia: Protagonismo e Inovações}


literatura, estudo de caso, textos incompletos ou que abordasse o TMI após o desmame do paciente na UTI, escore baixo e trabalhos com metodologia e/ou conclusões duvidosas.

A seleção dos materiais foi realizada em 3 etapas: a primeira caracterizada pela busca nas bases de dados, no período de outubro de 2019 à novembro de 2021, sendo selecionados 30 artigos, a segunda etapa caracterizada pela leitura do título e resumo, excluindo 25 artigos os quais não tinham relevância com o tema e não atendiam integralmente aos critérios de inclusão; na terceira etapa, após leitura na íntegra dos textos disponíveis, foi escolhido 5 trabalhos para compor esta revisão.

Tabela 1: Características dos trabalhos originais selecionados, publicados entre 2017 a 2021.

\begin{tabular}{|c|c|c|c|c|c|}
\hline AUTOR & $\begin{array}{l}\text { TIPO DE } \\
\text { ESTUDO }\end{array}$ & $\begin{array}{c}\text { DESCRIÇÃO } \\
\text { DA } \\
\text { AMOSTRA }\end{array}$ & $\begin{array}{l}\text { INSTRUMENTOS } \\
\text { DE COLETA }\end{array}$ & INTERVENÇÃO & $\begin{array}{l}\text { PRINCIPAIS } \\
\text { RESULTADOS }\end{array}$ \\
\hline $\begin{array}{l}\text { MORENO } \\
\text { et.al, } \\
2019\end{array}$ & $\begin{array}{l}\text { Ensaio } \\
\text { clínico } \\
\text { controlado } \\
\text { randomizado, } \\
\text { duplo-cego }\end{array}$ & $\begin{array}{l}126 \text { pacientes } \\
\text { em ventilação } \\
\text { mecânica por } \\
48 \text { horas ou } \\
\text { mais }\end{array}$ & $\begin{array}{l}\text { Manuvacuômetro } \\
\text { digital Carefusión }\end{array}$ & $\begin{array}{l}\text { Manuvacuometria } \\
\text { em ambos os } \\
\text { grupos. Grupo } \\
\text { Controle recebeu } \\
\text { Tratamento } \\
\text { convencional } \\
\text { (fisioterapia } \\
\text { respiratória e } \\
\text { manejo da VM) e } \\
\text { o Grupo } \\
\text { Experimental } \\
\text { recebeu } \\
\text { tratamento } \\
\text { adicional com o } \\
\text { programa de EMR } \\
\text { conduzido com o } \\
\text { IMT Threshold, } \\
\text { todos os dias, } 2 \\
\text { vezes ao dia, } 3 \\
\text { séries de } 6 \text { a } 10 \\
\text { repetições cada, } \\
\text { com } 2 \text { minutos de } \\
\text { descanso entre } \\
\text { cada série. } \\
\text { A carga inicial do } \\
\text { treinamento foi } \\
\text { ajustado } \\
\text { considerando } 50 \% \\
\text { da } \\
\text { inspiratória pressão } \\
\text { máxima (PImáx). }\end{array}$ & $\begin{array}{l}\text { Não houve } \\
\text { diferenças } \\
\text { estatisticamente } \\
\text { significantes na } \\
\text { mediana de } \\
\text { tempo } \\
\text { desmame da VM } \\
\text { entre os grupos ou } \\
\text { a probabilidade de } \\
\text { extubação entre os } \\
\text { grupos } \\
\text { (HR: 0,82; IC } \\
95 \% \text { : } 0,55-1,20 ; p \\
=0,29) \text {. A pressão } \\
\text { inspiratória } \\
\text { máxima aumentou } \\
\text { no } \\
\text { grupo } \\
\text { experimental em } \\
\text { média } 9,43 \\
(17,48) \mathrm{cm} H 2 \mathrm{H} \text { e } \\
\text { no convencional } \\
5,92 \quad(11,90) \mathrm{cm} \\
\mathrm{H} 2 \mathrm{O} \\
(\mathrm{p}=0,48) .\end{array}$ \\
\hline $\begin{array}{l}\text { TONELLA } \\
\text { et.al, } \\
2017\end{array}$ & $\begin{array}{l}\text { Estudo } \\
\text { prospectivo } \\
\text { randomizado }\end{array}$ & $\begin{array}{lr}21 & \text { pacientes } \\
\text { randomizados } \\
\text { em } & \text { dois } \\
\text { grupos: } & 11 \text { no }\end{array}$ & $\begin{array}{l}\text { Mensuração da } \\
\text { PIMax através do } \\
\text { Manovacuômetro } \\
\text { digital }\end{array}$ & $\begin{array}{lr}\text { No grupo } & \text { INP } \\
\text { seguindo } & \text { o } \\
\text { protocolo } & \\
\text { institucional, } & \text { os } \\
\end{array}$ & $\begin{array}{l}\text { No grupo EIMT, a } \\
\text { pressão } \\
\text { inspiratória } \\
\text { máxima (PImáx) }\end{array}$ \\
\hline
\end{tabular}
Inovações 


\begin{tabular}{|c|c|c|c|c|c|}
\hline & & $\begin{array}{l}\text { INP (controle) } \\
\text { e } 10 \text { no EIMT } \\
\text { (intervenção) }\end{array}$ & $\begin{array}{l}\text { GlobalMed }^{\circledR} ; \\
\text { Índice de } \\
\text { Respiração Rápida e } \\
\text { Superficial (RSBI) } \\
\text { com o ventilômetro } \\
\text { Wright }{ }^{\circledR} \text { Mark-8. }\end{array}$ & $\begin{array}{l}\text { pacientes foram } \\
\text { submetidos a um } \\
\text { INP usando uma } \\
\text { peça em T por uma } \\
\text { duração } \\
\text { progressivamente } \\
\text { crescente, até } \\
\text { completar } \\
\text { horas } \\
\text { autonomia } \\
\text { respiratória em } \\
\text { nebulização } \\
\text { contínua. No } \\
\text { grupo EIMT, os } \\
\text { pacientes usaram } \\
\text { um dispositivo de } \\
\text { treinamento } \\
\text { inspiratório } \\
\text { eletrônicoPOWER } \\
\text { respirar }{ }^{\circ} \text { KH2, } \\
\text { realizando } 3 \text { séries } \\
\text { de 10 com } \\
\text { intervalo de } 1 \\
\text { minuto entre cada } \\
\text { séries, duas vezes } \\
\text { ao dia, com carga } \\
\text { resistiva de } 30 \% \\
\text { da PImáx inicial e } \\
\text { reajustada } \\
\text { diariamente, com } \\
\text { um aumento de } \\
10 \% \text { em cada dia } \\
\text { de treinamento. }\end{array}$ & $\begin{array}{lr}\text { após } & \text { o } \\
\text { treinamento foi } \\
\text { significativamente } \\
\text { maior que a } \\
\text { anterior (P = } \\
\text { 0,017), não houve } \\
\text { alterações } \\
\text { hemodinâmicas e } \\
\text { o tempo total de } \\
\text { desmame foi } \\
\text { menor que no } \\
\text { grupo INP (P = } \\
0,0192) \text {. }\end{array}$ \\
\hline $\begin{array}{l}\text { PASCOTINI } \\
\text { et.al, } \\
2017\end{array}$ & $\begin{array}{l}\text { Experimental } \\
\text { do tipo pré e } \\
\text { pós- } \\
\text { intervenção, } \\
\text { com análise } \\
\text { quantitativa } \\
\text { dos dados. }\end{array}$ & $\begin{array}{l}14 \text { pacientes } \\
\text { (três do gênero } \\
\text { masculino e } \\
\text { onze do gênero } \\
\text { feminino), } \\
\text { distribuídos } \\
\text { aleatoriamente } \\
\text { em Grupo } \\
\text { Controle (GI) } \\
\text { e Grupo } \\
\text { Experimental } \\
\text { (GII) }\end{array}$ & $\begin{array}{l}\text { Manovacuômetro } \\
\text { analógico (Suporte } \\
\text { R) }\end{array}$ & $\begin{array}{l}\text { Diariamente, } \\
\text { durante sete dias, o } \\
\text { GI recebeu três } \\
\text { sessões de } \\
\text { fisioterapia } \\
\text { convencional e o } \\
\text { GII realizou, } \\
\text { adicionalmente, } \\
\text { treinamento } \\
\text { muscular } \\
\text { respiratório } \\
\text { (TMR) com o } \\
\text { Threshold IMT®, } \\
\text { uma vez ao dia, no } \\
\text { período da tarde, } \\
\text { conectado à } \\
\text { traqueostomia, } \\
\text { sendo três séries } \\
\text { de dez repetições } \\
\text { com carga de } \\
20 \% \text { da PImáx. }\end{array}$ & $\begin{array}{l}\text { Observou-se } \\
\text { aumento (p=0,02) } \\
\text { na FR e redução } \\
\text { da PImáx } \\
\text { (p=0,04) no GI, } \\
\text { demonstrando } \\
\text { aumento do } \\
\text { trabalho } \\
\text { respiratório e } \\
\text { perda de força } \\
\text { muscular entre o } \\
\text { primeiro e sétimo } \\
\text { dia de desmame. } \\
\text { No GII, as } \\
\text { variáveis } \\
\text { não sofreram } \\
\text { alterações } \\
\text { significativas, } \\
\text { observando-se a } \\
\text { manutenção da } \\
\text { função } \\
\text { respiratória }\end{array}$ \\
\hline $\begin{array}{l}\text { HELBOUHY } \\
\text { et.al, } \\
2018\end{array}$ & $\begin{array}{l}\text { Ensaio } \\
\text { clínico } \\
\text { controlado }\end{array}$ & $\begin{array}{lr}40 & \text { pacientes } \\
\text { com } & \text { DPOC } \\
\text { dividos } & \text { em } \\
\text { Grupo } & \text { (A) }\end{array}$ & $\begin{array}{l}\text { Ventilador Modelo } \\
\text { Servo I maquet e } \\
\text { ventilador Modelo } \\
\text { Servo } 300\end{array}$ & $\begin{array}{l}\text { O grupo (A) foi } \\
\text { treinado baseado } \\
\text { na diminuição } \\
\text { gradual } \quad \text { da }\end{array}$ & $\begin{array}{l}\text { Houve uma } \\
\text { diferença } \\
\text { significativa entre } \\
\text { os dois grupos em }\end{array}$ \\
\hline
\end{tabular}




\begin{tabular}{|c|c|c|c|c|c|}
\hline & & $\begin{array}{l}\text { experimental e } \\
\text { Grupo (B) } \\
\text { controle }\end{array}$ & & 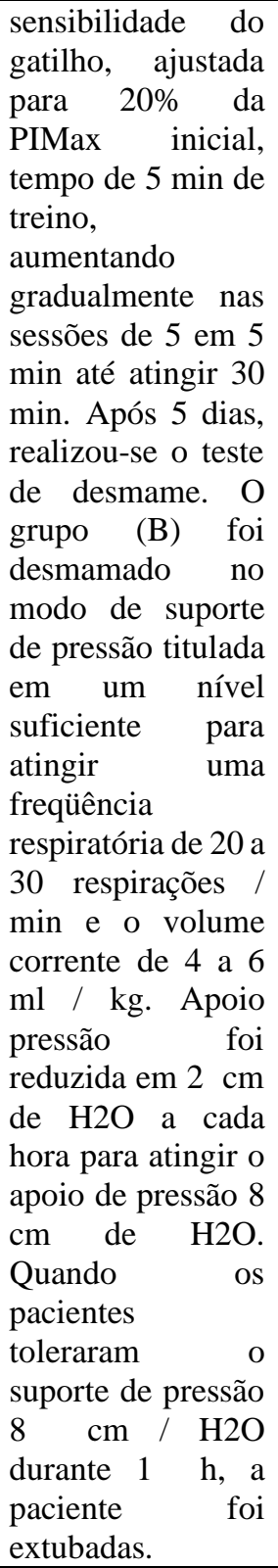 & $\begin{array}{l}\text { relação a taxa de } \\
\text { sucesso no } \\
\text { desmame, duração } \\
\text { da ventilação } \\
\text { mecânica, tempo } \\
\text { de permanência na } \\
\text { UTI, tempo de } \\
\text { internação. } \\
\text { Também houve } \\
\text { uma melhora } \\
\text { significativa no } \\
\text { grupo (A), } \\
\text { incluindo PO2, } \\
\text { saturação de O2, } \\
\text { TV, RR, MIP ao } \\
\text { longo dos } 5 \text { dias } \\
\text { de TMI. }\end{array}$ \\
\hline $\begin{array}{l}\text { CONDESSA } \\
\text { et.al, } \\
2019\end{array}$ & $\begin{array}{l}\text { Ensaio } \\
\text { clínico } \\
\text { controlado } \\
\text { randomizado }\end{array}$ & 92 pacientes & Ventilômetro & $\begin{array}{l}\text { O grupo } \\
\text { experimental } \\
\text { recebeu cuidados } \\
\text { usuais e } \\
\text { treinamento } \\
\text { muscular } \\
\text { inspiratório } \\
\text { usando um } \\
\text { dispositivo de } \\
\text { limiar, com uma } \\
\text { carga de } 40 \% \text { de } \\
\text { sua pressão } \\
\text { inspiratória } \\
\text { máxima, com um } \\
\text { regime de } 5 \text { séries } \\
\text { de } 10 \text { respirações, } \\
\text { duas vezes por } \\
\text { dia, } 7 \text { dias por } \\
\text { semana. O grupo }\end{array}$ & $\begin{array}{l}\text { Observou- se que } \\
\text { houve um período } \\
\text { de desmame } 8 \\
\text { horas mais curto } \\
\text { no grupo } \\
\text { experimental, } \\
\text { embora não foi } \\
\text { estatisticamente } \\
\text { significativo. As } \\
\text { pressões } \\
\text { inspiratórias e } \\
\text { expiratórias } \\
\text { máximas } \\
\text { aumentaram no } \\
\text { grupo } \\
\text { experimental e } \\
\text { diminuíram no } \\
\text { grupo controle, } \\
\text { com diferenças }\end{array}$ \\
\hline
\end{tabular}




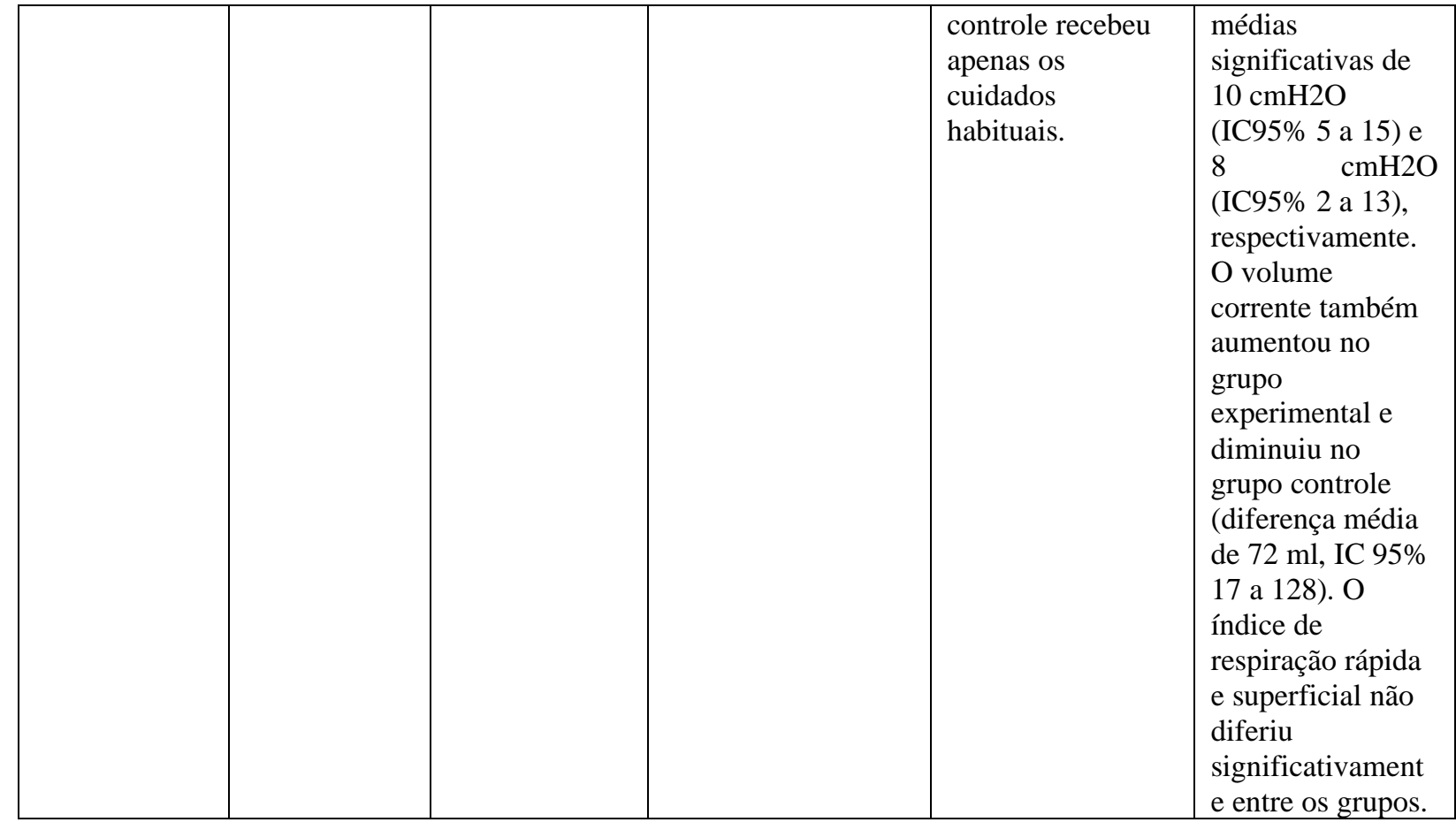

\section{CONCLUSÃO}

De acordo com os resultados encontrados, foi reconhecido que quanto maior a força dos musculos respiratorios, maior as chances de sucesso no desmame, consequentemente menor será o tempo de permanência na UTI e prevenção de complicações associadas à internação hospitalar prolongada.

\section{REFERÊNCIAS}

CONDESSA, Robledo L. et al. Inspiratory muscle training did not accelerate weaning from mechanical ventilation but did improve tidal volume and maximal respiratory pressures: a randomised trial. Journal of physiotherapy, v. 59, n. 2, p. 101-107, 2017.

PASCOTINI, S. et al. Respiratory muscle training in patients weaning from mechanical ventilation. ABCS Health Sci, v. 39, p. 12-16, 2018.

ELBOUHY, Mohammed S.; ABDELHALIM, Hesham A.; HASHEM, Ahmed MA. Efeito do treinamento dos músculos respiratórios no desmame de pacientes com DPOC ventilados mecanicamente. Jornal Egípcio de Doenças Torácicas e Tuberculose, v. 63, n. 3, p. 679-687, 2018.

TONELLA, Rodrigo Marques et al. Inspiratory muscle training in the intensive care unit: a new perspective. Journal of clinical medicine research, v. 9, n. 11, p. 929, 2018. 
OFFMAN, Mariana et al. Can inspiratory muscle training improve weaning outcomes in difficult to wean patients? A protocol for a randomised controlled trial (IMweanT study). BMJ open, v. 8, n. 6, p. e021091, 2018.

MORENO, LM Sandoval et al. Efficacy of respiratory muscle training in weaning of mechanical ventilation in patients with mechanical ventilation for 48 hours or more: A Randomized Controlled Clinical Trial. Medicina Intensiva (English Edition), v. 43, n. 2, p. 79-89, 2019.

VIANA, Yulle Christine Goiabeira et al. Treinamento Muscular Inspiratório no Pós-Operatório Cardíaco: Uma Revisão Sistemática/Inspirational Muscle Training in the Postoperative Cardiac: A Systematic Review. Saúde em Foco, p. 50-69, 2020. 